\title{
A training intervention to reduce paternalistic care and promote autonomy: a preliminary study
}

This article was published in the following Dove Press journal:

Clinical Interventions in Aging

\author{
Macarena Sánchez- \\ Izquierdo' \\ Marta Santacreu ${ }^{2}$ \\ Ricardo Olmos ${ }^{3}$ \\ Rocío Fernández- \\ Ballesteros ${ }^{4}$ \\ 'Department of Psychology, Universidad \\ Pontificia Comillas, Madrid 28049, Spain; \\ ${ }^{2}$ Department of Psychology, National \\ Distance Education University of Spain - \\ UNED, Madrid 28670, Spain; \\ ${ }^{3}$ Department of Methodology, \\ Autonomous University of Madrid, \\ Madrid 28049, Spain; ${ }^{4}$ Department of \\ Psychobiology and Health, Autonomous \\ University of Madrid, Madrid 28049, \\ Spain
}

Introduction: Paternalism, assuming control of aged care, is a widespread orientation in older adults care. Paternalistic attitudes and practices are commonly understood as a threat to the freedom and autonomy of a person, making patients more dependent. Therefore, the reduction of these attitudes and behaviors is a primary goal for any older adult health and social care situation. The aim of this preliminary study is to develop a behavioral intervention to decrease paternalistic behaviors in formal caregivers and to increase those care behaviors which promote autonomy at post-intervention (1 week) and at follow-up (14 weeks).

Methods: A sample of 118 professional caregiver volunteers working in day care centers and nursing homes were assigned to quasi-experimental $(\mathrm{N}=47)$ and control $(\mathrm{N}=71)$ conditions. The intervention consisted of 3 weekly group sessions. Individual and contextual measures were collected: 1) the primary outcome variable was the type of care (paternalistic versus autonomist) measured through the self-report Paternalist/Autonomist Care Assessment (PACA); 2) A 10-item caregiver self-register of paternalistic behaviors was carried out, 3) Finally, in order to assess the potential effects on observed behavior both in caregiver and older adult functioning at a contextual level, the five institutions were assessed through the SERA-RS.

Results: Compared with the control group, caregivers in the behavioral intervention group displayed significantly lower paternalistic appraisals at posttest and follow-up. Regarding the intervention group, caregivers at posttest and follow-up showed significantly greater occurrence of autonomist behaviors being promoted and lower paternalistic appraisal. The results regarding the effect on the institutions showed better personnel performance and older adult functioning.

Conclusion: Caregivers who followed the intervention learned to better identify older adult needs; although we did not find significant differences in autonomy occurrence compared with the control group, a behavioral intervention may promote more autonomist environments and, therefore, better personnel and older adult functioning.

Keywords: paternalism, autonomy, caregivers, behavioral intervention

\section{Introduction}

Taking care of older adults is a complex and multifaceted task in which an older adult interacts with caregivers (family members and/or professionals), in a particular context, receiving support when needed (unfortunately, sometimes also when not needed). The appropriate level and type of care must be chosen from a very diverse range (medical, physical, psychological, or social, among others), and the nature of care provided not only depends on the care required by the older adult's condition but also on the caregiver's knowledge, caring abilities, and views of aging.
Correspondence: Macarena SánchezIzquierdo

Department of Psychology, Comillas Pontifical University, C. Universidad Comillas, 3-5, Madrid 28049, Spain Tel +349l 7343950

Email msizquierdo@comillas.edu 
In this complex human situation, two main antagonistic perspectives can be identified: paternalistic (caregiver centered), under which caregivers of older adults make decisions in another person's best interests, without that person's request, consent, or awareness of that person's welfare; ${ }^{1,2}$ autonomist (person/care-recipient centered), where caregivers reinforce individual choice, the self-government and independence/autonomy of the care recipient. ${ }^{3,4}$

Paternalist care is an attitude in which the caregiver is considered above the care recipient, that is, a traditional "institutional" approach. ${ }^{5}$ The care recipients are considered to play a passive role. This position is mainly held by clinicians, who expect to make the best decision for their patients. Family members also usually approach their care recipients from this perspective, wanting the best for their loved ones but sometimes underestimating their capacity and wishes. ${ }^{6}$ Likewise, aged-care centers tend to be places dominated by the biomedical model and hierarchical decisionmaking, ${ }^{5,7,8}$ where values such as efficiency and security are paramount. In these contexts, older adults are usually treated as children, in need of security and overprotection, through the use of childish language with plenty of diminutives, and even with excessive signs of affection because of their illness, disability or handicaps. ${ }^{8-10}$ In this context, the autonomist type of care is usually considered to be "less safe", 5 showing higher rate of falls in older adults in person-centered care compared with older adults under traditional aged care. ${ }^{11}$ Thus, research has shown that paternalistic care of older adults is associated with a decrease in older adult autonomy. ${ }^{12-15}$

Alternatively, with the aim of promoting autonomy, another social and health care approach is emerging: person-centered care. Several studies have shown the importance of maintaining a relationship with the older adult in which autonomy, independence, respect, and choice replace disempowerment, overprotection, and stigmatization. ${ }^{16-18}$ Even when older adults are tested by asking them how they want to be treated, their answers are: "as adults (not as children)", "being able to choose", "with dignity and respect", "being informed and asked" ${ }^{19,20}$ In sum, all these remarks and preferences correspond to an "autonomist" care model which is "person-centered" and promotes the autonomy of older adult care recipients, ${ }^{21}$ ensuring patient safety whilst always seeking to provide care that is truly person-centered. ${ }^{22}$

This standpoint, from the ideal of an egalitarian caregiver-patient relationship, ${ }^{23}$ promotes the inclusion of the patient in the caregiver's decision-making process ${ }^{24}$ on the basis that care recipients are key elements in the selection of the best care options (see also ${ }^{25}$ ), emphasizing the wellbeing and quality of life as defined by the individual. ${ }^{17}$

Although there is a growing body of literature on person-centered care, there is no consensus on its definition, and therefore, in its key elements and measures to assess effectiveness. ${ }^{4}$ The American Geriatrics Society Expert Panel on Person-Centered Care has attempted to unify the different definitions, proposing the following definition:

\begin{abstract}
"Person-centered care" means that individuals' values and preferences are elicited and, once expressed, guide all aspects of their health care, supporting their realistic health and life goals. Person-centered care is achieved through a dynamic relationship among individuals, others who are important to them, and all relevant providers. This collaboration informs decision-making to the extent that the individual desires ${ }^{4}$ p.16)
\end{abstract}

Person-centered care includes the following elements: personhood, ${ }^{26}$ knowing the person, ${ }^{27}$ maximizing choice and autonomy, ${ }^{23}$ quality care, ${ }^{27,28}$ nurturing relationships, ${ }^{26,29}$ and a supportive physical and organizational environment. ${ }^{18,30}$

Several studies have shown that this care approach improves the professional's capacity to meet individual needs with dignity and respect, increases social interaction between residents, ${ }^{31}$ improves the psychological status, ${ }^{32,33}$ physical and psychological health ${ }^{33}$ of older adults, increases patient satisfaction, ${ }^{34,35}$ promotes older-adults' views about quality of care ${ }^{36,37}$ and even improves the continuity of residents' care $^{38}$ and staff satisfaction. ${ }^{31,38-40}$ Furthermore, International Institutes have highlighted the need for this approach to care (eg, the World Health Organization, ${ }^{41}$ the Health Foundation ${ }^{42,43}$ and the Institute of Medicine ${ }^{44}$ ).

Several factors can lead to the type of care provided, such as age, functional conditions, state of awareness, legal situation, etc. Lawton and Nahemow ${ }^{45}$ pointed out this position in the core of their person/environment interactional theory. In this theory, they posited that the type of care provided to older adults is the result of interaction between two factors: the level of the older adult's competence, frailty, dependency on the one hand and/or cognitive impairment on the other, both of which are mediated by environmental pressures. Giving information to patients, reinforcing older adults' autonomy and involving them in the decision-making process have also been highlighted. ${ }^{25,46-48}$ 
Despite this, it seems obvious that the client's competence should be the foremost consideration in deciding the level of support provided in care, ${ }^{49,50}$ this, however, is not common practice. ${ }^{42,51}$ Clearly, it should be underlined that, depending on the condition of the older person, they may require care, support, company, protection, or none of these at all. However, caregivers often undervalue the older person's abilities, ${ }^{12-15}$ thereby following stereotypes; they do not treat them as adults, they provide unnecessary help and attempt to restrict their activities. When caregivers overprotect care recipients who do not ask for, nor need, help and do not require protection, autonomy may be reduced; this constitutes the paternalistic approach. ${ }^{12-15}$

As noted above, paternalistic care reduces the older adult's autonomy, and studies have found widespread paternalistic care in formal contexts; thus developing effective interventions to promote autonomist care is a priority. In their systematic review, Brownie and Nancarrow ${ }^{52}$ highlight the need to emphasize the effectiveness of the elements of person-centered care, either singly or in combination. There have been several interventions along such lines which, although not focusing on paternalistic behaviors, have tried to improve several aspects of resident and staff functioning. For example, several interventions focused on caregiver/patient communication/interactions and dementia care ( $\mathrm{se}^{53,54}$ for examples) showed that the intervention improved communication between staff and residents during care routines, increasing use of positive statements and not requiring more staff time for the daily activities, but were unable to affect older adults' verbal interactions with professionals or their disruptive behavior. Other studies have focused on improving communication among older adults, nursing staff, and visiting family members, ${ }^{55,56}$ which had beneficial effects on residents (depression, irritability, and verbal behavior) but not on the nursing staff's management of problem behaviors.

Two main findings highlight the need to study how to change paternalistic behaviors to promote autonomy: 1) care professionals who hold negative age stereotypes provide paternalistic care to older adults; ${ }^{57}$ and 2) caregiver functioning influences older adult functioning; that is, when caregivers consider older adults as dependent, it becomes a self-fulfilling prophecy., ${ }^{3,13,58}$

Changing stereotypes is increasingly taken into consideration as a critical component of healthy aging. ${ }^{59}$ Although few studies have tested the effectiveness of interventions to reduce or prevent ageist stereotypes, our cross-cultural results $^{60}$ yielded positive effects of university programs, not only in personal views about ageing, but in cultural stereotypes. From a behavioral perspective, several studies show effective results in changing thoughts and action through traditional behavioral techniques, such as modeling, shaping and reinforcement. ${ }^{61}$

The aim of the present study is to examine the effect of a behavioral group intervention in day care centers and nursing homes to decrease professional caregivers' paternalistic behaviors and to encourage those behaviors that promote autonomy in older adults. Moreover, we assess the potential effects caused by our behavioral intervention in the institutions where the intervention was implemented. Therefore, our hypotheses are:

1. Those caregivers following the behavioral intervention will report significantly lower appraisal of paternalistic behaviors and greater appraisal of autonomist behaviors, measured by the Paternalist/Autonomist Care Assessment (PACA).

2. Those caregivers following the behavioral intervention will observe the occurrence in their settings of significantly fewer paternalistic behaviors and more behaviors promoting autonomy, measured by the Paternalist/Autonomist Care Assessment (PACA).

3. The institutions in which the intervention was implemented will show a significant improvement in professional functioning and older care recipients, measured by MEAP/SERA-RS.

\section{Method}

\section{Procedure}

Participants were recruited through different centers (day care centers and nursing homes) of a private non-profit older adult services company, ASISPA. The researchers contacted the director by telephone and visited the company, subsequently doing the same with the directors of the different older adult centers; they explained the general objectives of the study and requested permission to implement the intervention. If participation was agreed, one of the researchers visited the center, explained the general objectives of the study to the professionals and created the intervention group with those professionals who were interested in taking part.

The professionals working in the day care center/nursing home who did not want to participate in the intervention group were asked to complete the questionnaires voluntarily, becoming the control group. Participants in 
the intervention and control conditions were therefore not randomly assigned.

\section{Ethical considerations}

This study was approved by the Ethics Committee of the Autonomous University of Madrid (November 2014). All professionals who participated, either in the experimental or in the control group, gave their written informed consent to participate prior to the study's start date and were also informed about how the study could help to improve care as well as the possibility of dropping out of the study with no negative consequences at any time. The directors of each center also gave their explicit authorization to collect information in the institutions. Data were analyzed anonymously.

\section{Participants}

The sample was made up of professional caregivers working with older adults in two contexts $(\mathrm{N}=120)$ : three day care centers (DCCs) in Madrid city $(\mathrm{N}=54)$ and two nursing homes (NH) in Madrid region $(\mathrm{N}=64)$ (Spain). All these centers are managed by ASISPA (a private non-profit older adult services company) (Table 1).

Some professional caregivers from these centers voluntarily participated in weekly behavioral group intervention sessions during a total of three weeks (intervention group). As already noted above, some of those caregivers that did not participate in the intervention were assigned to the control group. The intervention group was made up of 47 volunteers who were interested in taking part, 22 from two nursing homes $(83.39 \%$ women, mean age $=42.57$ years, $\mathrm{SD}=10.54$ years) and 25 from three day care centers $(66.67 \%$ women, mean age $=41.92$ years, $\mathrm{SD}=9.00$ years). Regarding their professions, most (85.7\%) were health professionals (psychologists, medical doctors, nurses, physiotherapists, social workers and other health professionals) and $14.3 \%$ had other unspecified professions.

Seventy-one caregivers working at the same centers who did not wish to participate were assigned to the control group $(75.3 \%$ women, mean age $=41.30$ years,

Table I Distribution of the sample

\begin{tabular}{|l|l|l|l|}
\hline & Nursing home & Day care center & \\
\hline Intervention group & 22 & 25 & 47 \\
Control group & 44 & 29 & 73 \\
& 66 & 54 & \\
\hline
\end{tabular}

$\mathrm{SD}=10.09$ years). Regarding their professions, the majority $(69.9 \%)$ were health professionals (medical doctors, nurses, auxiliary nurses, social workers and other health professionals) and $30.1 \%$ had other unspecified professions. No significant demographic differences were found between experimental and control groups.

In terms of participation in post and follow-up of experimental subjects, only $2.13 \%$ and $21.28 \%$ did not participate in the post-test and follow-up, respectively. Of the controls, $52.05 \%$ in the post-test and $19.18 \%$ in the follow-up did not complete the corresponding forms. The high attrition rate in the post-test of the control group was mainly due to a low degree of motivation among the participants to fill out the questionnaires. Because they were not interested in the study, they may have perceived the questionnaires as too much extra work. In the followup, this was improved through a motivational initiative carried out by the researcher and the center directors to obtain the maximum number of completed questionnaires.

\section{Instruments}

In order to assess hypothetical individual outcomes and potential impacts on the context after the implementation of the program, two types of measures were administered: individual and contextual.

Control and intervention groups both completed the Paternalist/Autonomist Care Assessment-PACA, with the intervention group also completing the self-monitoring register. MEAP/SERA-RS- Assessment system for older adult nursing homes was implemented in the five institutions before the behavioral intervention, and one week and 14 weeks afterwards, that is, during pre, post and followup phases, respectively.

The Paternalist/Autonomist Care Assessment $(P A C A) .{ }^{67}$ This self-report assesses the appraisal and occurrence of 30 statements about two ways of treating older adults ("paternalistic" and "autonomist") on a fourpoint Likert-type scale asking the extent of agreement with a given item. The instructions are as follows:

In the first column of this questionnaire, you can find 30 statements describing forms of treating or caring for older adults. In the second column, please score to what extent you agree with each statement by circling a number from 1 (do not agree at all) to 4 (totally agree). In the third column, please circle YES or NO whether each statement occurs in your center. 
The two sub-scales showed good construct and concurrent empirical validity, internal consistency $(\alpha=0.926$ "paternalist" items, and $\alpha=0.726$ "autonomist" items) and convergent and discriminant validity (69). The PACA also measures whether each statement occurs in the specific institution where the caregiver works, with a yes/no question, which again yielded good construct and concurrent empirical validity, internal consistency $(\alpha=0.913$ paternalist items and $\alpha=0.756$ autonomist items) and convergent and discriminant validity. ${ }^{68}$

Caregivers' Self-register of their own paternalistic behaviors. This is a self-register of the frequency of the ten most representative paternalistic behaviors: 1) Using childish language, 2) Grooming and excessive touching of recipients; 3) Loud or exaggerated tone even when there are no signs of hearing problems; 4). Ignoring the older client, speaking to the accompanying relative; 5) Ignoring the older adult's presence and talking about him/her to the family caregivers or other staff; 6) Finishing the older adult's sentences; 7) Not explaining what he/she is going to do, nor the daily activities; 8) Doing or finishing a task without asking whether he/she needs help with it; 9) Lack of privacy in their personal hygiene, and 10) Any other behavior that caregivers may detect. Caregivers were asked to register the frequency of these paternalistic behaviors daily for two consecutive weeks after session two and three of the intervention.

Assessment system for older adult nursing homes (MEAP/ SERA-RS; ${ }^{69}$ adapted from the MEAP,$\left.{ }^{70}\right)$. The instrument is completed by an external observer reporting older adult and professional functioning on a scale of 0 to 3 . The two subscales included were 1) Older adult functioning scale, which includes five observational items: personal grooming, condition of clothing, older adult interaction, brief verbal exchanges, and general activity level $(\alpha=0.76)$, and 2$)$ Personnel functioning scale, which includes five observational items: quality of interaction, physical contact with older adults, organization, caregiver availability for older adults (users or residents), and caregiver conflict $(\alpha=0.92)$.

\section{The intervention}

There were 7 intervention groups, ranging from 5 to 9 participants. Each center had one experimental/intervention group, except in one nursing home and one day care center where two intervention/experimental groups were involved, respectively. Each intervention group followed a behavioral intervention, consisting of $60 \mathrm{~min}$ sessions once a week for three weeks. Details of the program are shown in Table 2.

The intervention was based on behavioral knowledge and on the principles of behavioral therapy, and was implemented by a trained psychologist. We designed it to teach professional caregivers how to identify paternalistic behaviors and change them into autonomist ones, using a variety of different behavioral techniques: 1) learning as a model, 2) role-playing as a tool to help caregivers identify if paternalistic behaviors were involved, 3) modeling to perform alternative behaviors, and 4) reinforcing autonomist behaviors shown by participants.

Table 2 Schedule

\begin{tabular}{|c|c|c|c|c|}
\hline Module & Sessions & Timing & Objectives & Intervention components \\
\hline Pre-test & I & Ist week & & PACA, MEAP/SERA-RS \\
\hline $\begin{array}{l}\text { Module I. Identifying } \\
\text { paternalistic behaviors }\end{array}$ & I & 2nd week & To identify paternalistic behaviors. & Education, debate \\
\hline $\begin{array}{l}\text { Module } 2 \text {. Identifying } \\
\text { paternalistic behaviors }\end{array}$ & 1 & $3 r d$ week & $\begin{array}{l}\text { To learn how to identify caregivers' } \\
\text { paternalistic behaviors and in which } \\
\text { situations they are more likely to } \\
\text { appear. }\end{array}$ & $\begin{array}{l}\text { Education, role-play and discussion } \\
\text { Instrument administered: self- } \\
\text { monitoring paternalistic behaviors }\end{array}$ \\
\hline $\begin{array}{l}\text { Module } 3 \text {. Identifying and } \\
\text { molding autonomist } \\
\text { behaviors. Artificial } \\
\text { situation }\end{array}$ & 1 & 4th week & $\begin{array}{l}\text { To learn and practice alternative ways of } \\
\text { behaving that promote older adult } \\
\text { autonomy. }\end{array}$ & $\begin{array}{l}\text { Role play, reinforcement of autonomist } \\
\text { behaviors shown by participants } \\
\text { (molding behavior); debate, discussion. } \\
\text { Instrument administered: self- } \\
\text { monitoring paternalistic behaviors }\end{array}$ \\
\hline Posttest and follow-up & 1 & 5th and I4th week & & PACA, MEAP/SERA-RS \\
\hline
\end{tabular}

Abbreviation: PACA, paternalist/autonomist care assessment. 


\section{Contents of each session}

Session I. Module I. Identifying paternalistic behaviors

The first session started with a theoretical introduction about stereotypes and their effects on both older adult and staff functioning. The following concepts were introduced: person/environment interactional theory posited by Lawton and Namehow, ${ }^{45}$ the Stereotype Content Model (SCM) proposed by Fiske et al, ${ }^{71}$ the mediation of cultural stereotypes on caregiver/older adult functioning, how older adults want to be treated, and a final debate.

\section{Session 2. Module 2. Identifying paternalistic behaviors}

Session 2 started with a brief theoretical overview of stereotypes, behavioral prejudice, and their effects on older adults. The aim of the session was for participants to identify their own paternalistic behaviors when taking care of older adults; therefore, after an initial explanation, volunteers were asked to play the role of caregivers in a given common situation in older adult institutions (for example, taking a resident with functional motor problems from their bed to another room, hygiene actions, dressing, pushing wheelchairs, etc.). The remaining participants observed the role play and identified paternalistic behaviors (for example, moving the person without explaining where they were going, using childish vocabulary, and giving excess support, among others). The session ended with a discussion about common paternalistic behaviors by caregivers, their possible consequences, and possible alternative autonomist behaviors which could promote older adult autonomy. Participants were asked to self-monitor paternalistic behaviors in their daily work during the following week.

\section{Instruments administered: self-register}

Session 3. Module 3. Identifying and molding behavior. Promoting autonomist behaviors. Artificial situation

The trained psychologist guided caregivers' behaviors during the role play. Participants were shown how to perform alternative behaviors, and autonomist behaviors were reinforced. The session ended with a final conclusion about the benefits from autonomist behaviors and the possible harm caused by paternalistic behaviors, and a final debate about their intention to reinforce autonomist behaviors and the way to do it. Participants were asked to apply new selfmonitoring paternalistic behaviors during the following week.

Instruments administered: self-register

\section{Data analysis}

To evaluate the effects of the behavioral intervention over one and 14 weeks, a linear mixed model was applied, using SPSS v23 software with significance level at $p<0.05$ (two-tailed). The estimation method for parameters was Restricted Maximum Likelihood (REML). The independent variables were the intergroup experimental vs control condition, and the within-moment condition: pre, post and follow-up moments. Subjects were included as a random effect. The dependent variables were autonomy $v s$ paternalistic appraisal and occurrence (PACA), personnel and older adult functioning (MEAP/SERA-RS), and frequency of paternalistic behaviors (self-monitoring). For post hoc analysis, simple effects were examined in the linear mixed models. Missing data was treated with maximum likelihood estimation method (REML); this follows modern guidelines for missing data (eg, Enders, 2010). Finally, to analyze results in care center groups, independent $T$-test and ANOVA were conducted.

\section{Results}

Firstly, four mixed linear models were used to analyze the intergroup experimental and control condition in the within-moment condition (pre, post and follow-up tests). The dependent variables were the four measures from the Paternalist/Autonomist Care Assessment (PACA), that is, both appraisal of caregivers (Care Appraisal Scale- PACAAppraisal) and occurrence of behaviors (Occurrence of Care in Context- PACA-Occurrence).

Given the quasi-experimental nature of the study (nonrandom assignment to experimental conditions), two dependent variables showed significant differences between control and intervention group in the pre-test measures in particular: in the pre-test phase, the intervention group perceived a significantly lower occurrence of paternalistic behavior than the control group in its center $(p=0.043)$ and higher agreement with autonomist behaviors $(p=0.006)$ in terms of the way older adults should be treated $(p=0.006)$. Thus, it was more appropriate to study whether experimental and control groups changed their views during the study. Table 3 shows the $F$-statistic, $p$-value and sample means in the four dependent variables for experimental and control groups to evaluate if significant change occurred during the intervention.

In the intervention group, significant differences were detected during the study in the paternalistic appraisal measurement. This group significantly reduced their 


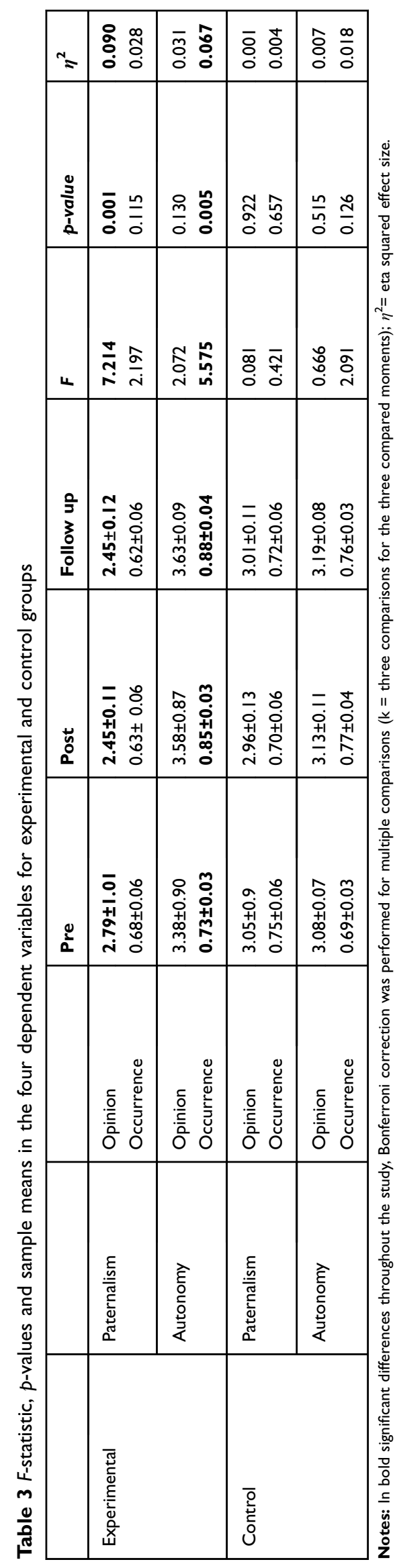

paternalistic appraisals of the way older adults should be treated in the study $\left(F(2,147)=7.214, p=0.001, \eta^{2}=0.090\right)$. Eta squared showed greater-than-medium effect size (Cohen, 1988) for this result. This did not happen in the control group, where there were no differences across the study $(F(2,211)=0.081, p=0.922)$.

In the perceived occurrence of paternalistic behavior, neither experimental nor control groups showed differences during the study ( $p>0.05)$. Finally, in the intervention group, a medium effect size and significantly higher scores were found for the perceived occurrence of autonomy in their centers during the study $(F(2,154)=5.575$, $\left.p=0.005 ; \eta^{2}=0.067\right)$, but this did not happen in the control group $(F(2,211)=2.091, p=0.126)$. Non-significant differences were not found throughout the study in either experimental or control groups regarding autonomist appraisals about how older people should be treated. Therefore, two measures were sensitive to the intervention study: paternalistic appraisal and occurrence of autonomy, and the changes occurred only in the intervention group.

Secondly, we studied whether significant changes occurred at care center level (contextual), with an external observer monitoring whether or not professional or older adult functioning improved. Thus, the effect of our behavioral intervention was examined in the five older adult institutions where the intervention was implemented. Mean scores showed a significant increase in positive personnel functioning at posttest and follow up $(p=0.009)$, and in older adult functioning at posttest and follow up $(p=0.004)$, measured by MEAP/SERA-RS (see Table 4).

\section{Discussion}

Beyond existing interventions for improving caregiver/ patient communication/interactions, the present study is a first attempt to develop a behavioral intervention for the reduction of paternalistic behaviors as well as the promotion of autonomy and independent behaviors in Spanish older adult settings. The main assumptions are that after our behavioral intervention, older adult caregivers would report less paternalistic appraisals regarding methods of care and more autonomist appraisals. This would have the following effects on the older adult institutions where the behavioral program was implemented: firstly, a reduction in the observed occurrence of paternalistic behaviors and an increased occurrence of observed autonomist behaviors. Secondly, improvements in professional carer functioning in: 1) their interactions with older adults (quality of 
Table 4 Means differences in older adult functioning and personnel functioning in five institutions

\begin{tabular}{|l|l|l|l|l|l|l|}
\hline & & Pre & Post & Follow up & $\boldsymbol{F}$ & $\boldsymbol{P}$-value \\
\hline \multirow{2}{*}{ Experimental } & Personnel functioning & $2.45 \pm 0.32$ & $2.88 \pm 0.27$ & $3.00 \pm 0.00$ & 7.189 & 0.009 \\
\cline { 2 - 7 } & Older adults functioning & $1.72 \pm 0.52$ & $2.16 \pm 0.33$ & $2.36 \pm 0.26$ & 3.589 & 0.004 \\
\hline
\end{tabular}

interaction, physical contact and caregiver availability for residents) 2) improvements in the organization of the care setting; and 3) a reduction in caregiver conflict. And finally, improvements in older adult functioning in: 1) their interactions with other older adults (older adult interaction, verbal exchanges), 2) general activity level, 3) personal grooming and 4) condition of clothing.

As expected, the data collected show differences in caregivers' paternalistic appraisal following behavioral intervention $(p=0.035)$. A post hoc explanation may be that caregivers who followed the intervention learned to better identify older adult needs and focus more on what the person could do, rather than on his/her disabilities. As Crandall highlighted, ${ }^{17}$ autonomist care requires caregivers to plan with the older adults, "determining the perspective of the person receiving care", and "to provide that assistance in such a way that clients are honored and valued and are not lost in the tasks of caregiving” (p. 48). As Fernández-Ballesteros et $\mathrm{al}^{72}$ emphasize, formal caregivers must adapt their care behaviors to the older adult's level of functioning; thus the level of autonomy promotion will depend on the older adult's resources and will be adapted to each patient, and in the case of dementia, to the stage and level of autonomy the older adult still has.

Furthermore, members of the experimental groups showed an improvement of promoting autonomy behaviors in the care setting $(p \leq 001)$. Hence, paternalistic self-monitoring tended $(p=0.084)$ to lead to reduced paternalistic behaviors. Our results show that although the intervention reduced paternalistic appraisal, paternalistic behaviors seemed to be more difficult to change. In a systematic review and meta-analysis of interventions to reduce ageism against older people (in the form of negative stereotypes, prejudice, and discrimination), Burnes et $\mathrm{al}^{73}$ have shown that these interventions had a strong effect on attitudes but not on how people worked with older adults.

We did not find differences in promoting autonomy appraisals and observed paternalistic behaviors mainly because there were significant differences at the pre-test phase between both groups (experimental vs control), showing higher agreement with autonomy behaviors $(p=0.006)$ and lower rates of paternalistic behaviors observed $(p=0.043)$ in caregivers who followed the intervention. A possible reason may be that most participants already held positive appraisals of autonomy and this might have had a strong influence to self-select into the intervention group.

Weiner and Ronch ${ }^{74}$ described the culture change movement of long-term care to more autonomist as a process of making "long-term care less about care tasks and more about caring for people and the relationships between people" (p.13). Regarding the contextual analysis for evaluating the potential effects of our behavioral intervention on both caregiver and older adult functioning (assessed by the MEAP/SERA-RS), as hypothesized, there is an improvement in personnel functioning $(p=0.009)$ and in older adult functioning $(p=0.004)$ after the behavioral intervention. It seems that better professional caregiver functioning (less paternalistic and more autonomist care behaviors) influences older adult functioning and may promote older adult independence.

When caregivers reduce their paternalistic behavior, residents' choice, autonomy and independence are enhanced; our assumption is that these interactions between older adults and staff increases social interaction between residents, verbal exchanges and general activity level, thereby improving older adult functioning. In this regard, previous studies have shown that types of caregiving behavior may exert a substantial impact on older adult functioning, , $^{3,17,18,74}$ and several studies have even shown that person-centered care was associated with improvements in staff ability to provide highquality individualized care, ${ }^{5,38}$ with lower dependency ${ }^{75}$ and with increased resident autonomy. ${ }^{76}$

In this regard, the intervention programs of Kihlgren et $\mathrm{al}^{77-79}$ resulted in positive effects on residents and increased interactions between residents and staff, and even increased the staff's understanding of dementia residents, becoming more sensitive to older adults needs.

Salas et $\mathrm{al}^{80,81}$ indicated that team training had a positive effect on team functioning, providing better care to patients, although the authors highlighted the importance of including opportunities for active learning, modeling, and practice. 
From this preliminary study, four main methodological limitations can be described: 1) high attrition at post-test, which was resolved in the follow-up; 2) the lack of a control group in the context design; 3) sample size might also be a limitation - further interventions involving larger samples are thus required; 4) the lack of change in perceived occurrence of paternalistic behavior in the experimental condition, which may reflect in part a need for more robust training to change behaviors and develop requisite skills, beyond merely attitudinal change, 5) the sensitivity of the measures might not be suitable for identifying changes - thus selecting more sensitive measures would be recommended; and 6) caregivers in the intervention group might already have held positive appraisals of autonomy, which might have led to substantial self-selection into the intervention group.

Since the type of formal care has important implications for both older adults and professionals, further research is needed to develop interventions aimed at promoting autonomist care.

\section{Conclusion}

The current findings suggest that the behavioral intervention designed is effective for reducing paternalistic appraisals and their occurrence as assessed by the PACA in those caregivers who follow the intervention in the setting, although we did not find significant differences in autonomy occurrence compared with the control group. A behavioral intervention may promote more autonomist environments and, therefore, better personnel and older adult functioning.

\section{Acknowledgment}

This study has been supported by MINECO Project: PSSI2014-5264-P-ICESEN)

\section{Disclosure}

The authors report no conflicts of interest in this work.

\section{References}

1. Culver CM, Gert B. The justification of paternalism. In: Robison WL, Pritchard MS, editors. Medical Responsibility. NJ: Human Press; 1979:1-14.

2. Halper T. The double-edged sword: paternalism as a policy in the problems of aging. Milbank Q. 1980;58(3):472-499. doi:10.2307/ 3349735

3. Fernández-Ballesteros R, Bustillos A, Santacreu M, Schettini R, DíazVeiga $\mathrm{P}$, Huici $\mathrm{C}$. Is older adult care mediated by caregivers' cultural stereotypes? The role of competence and warmth attribution. Clin Interv Aging. 2016;11:545-552. doi:10.2147/CIA.S96235
4. American Geriatrics Society Expert Panel on Person-Centered Care. Person-centered care: a definition and essential elements. $J \mathrm{Am}$ Geriatr Soc. 2016;64(1):15-18. doi:10.1111/jgs.13866

5. Brownie S, Nancarrow S. Effects of person-centered care on residents and staff in aged-care facilities: a systematic review. Clin Interv Aging. 2013;1-10.

6. Gallagher-Thompson D, Coon DW, Rivera P, Powers D, Zeiss AM. Family Caregiving. In: Hersen M, Van Hasselt VB (Eds.)Handbook of Clinical Geropsychology. Boston (MA): Springer US; 1998:469493.

7. Parsons T. The Social System. Glencoe (IL): Free Press; 1951.

8. Rosher RB, Robinson S. Impact of the eden alternative on family satisfaction. J Am Med Dir Assoc. 2005;6(3):189-193. doi:10.1016/j. jamda.2005.03.005

9. Nelson TD (Ed). Ageism : Stereotyping and Prejudice against Older Persons. London, UK: MIT Press; 2002:372.

10. Nelson TD. Ageism: prejudice against our feared future self. $J$ Soc Issues. 2005;61(2):207-221. doi:10.1111/j.1540-4560.2005.00402.x

11. Coleman MT, Looney S, O’Brien J, Ziegler C, Pastorino CA, Turner C. The Eden alternative: findings after 1 year of implementation. $J$ Gerontol A Biol Sci Med Sci. 2002;57(7):M422-M427. doi:10.1093/ gerona/57.7.M422

12. Cimarolli VR, Boerner K, Reinhardt JP, Horowitz A. Perceived overprotection, instrumental support and rehabilitation use in elders with vision loss: A longitudinal perspective. Psychol Health. 2013;28 (4):369-383. doi:10.1080/08870446.2012.729835

13. Thompson SC, Sobolew-Shubin A. Overprotective relationships: a nonsupportive side of social networks. Basic Appl Soc Psych. 1993;14(3):363-383. doi:10.1207/s15324834basp1403_8

14. Thompson SC, Sobolew-Shubin A. Perceptions of overprotection in III adults1. J Appl Soc Psychol. 1993;23(2):85-97. doi:10.1111/ j.1559-1816.1993.tb01053.x

15. Thompson S, Galbraith M, Thomas C, Swan J, Vrungos S. Caregivers of stroke patient family members: behavioral and attitudinal indicators of overprotective care. Psychol Health. 2002;17 (3):297-312. doi:10.1080/08870440290029557

16. Nolan MR, Brown J, Davies S, Nolan J, Keady J The senses framework: improving care for older people through a relationship-centred approach. Getting research into practice (GRiP) Report No 2. 2006.

17. Crandall LG, White DL, Schuldheis S, Talerico KA. Initiating person-centered care practices in long-term care facilities. J Gerontol Nurs. 2007;33(11):47-56.

18. Rader J, Semradek J. Organizational culture and bathing practice. $J$ Soc Work Long-Term Care. 2003;2(3-4):269-283. doi:10.1300/ J181v02n03_04

19. Fernández-Ballesteros R, Sánchez-Izquierdo $M$, Olmos $R$, et al. Cultural stereotypes in care contexts. Clin Interv Aging. 2018;13. doi:10.2147/CIA.S169487

20. Fernandez-Ballesteros R. Buen Trato. Cómo Quieren Ser Tratadas Las Personas Mayores. In: Guía PRÁCTICA Para Favorecer El Buen Trato a Las Personas Mayores. Madrid: SEGG; 2011:15-24.

21. Kane RA. Definition, measurement, and correlates of quality of life in nursing homes: toward a reasonable practice, research, and policy agenda. Gerontologist. 2003;43(2):28-36. doi:10.1093/geront/43. suppl_2.28

22. Fawcett T, Josephine N, Rhynas SJ. Re-finding the 'human side' of human factors in nursing: helping student nurses to combine personcentred care with the rigours of patient safety. Nurse Educ Today. 2014;34(9):1238-1241. doi:10.1016/j.nedt.2014.05.002

23. Mead N, Bower P. Patient-centredness: a conceptual framework and review of the empirical literature. Soc Sci Med. 2000;51:1087-1110. doi:10.1016/S0277-9536(00)00098-8

24. Leplege A, Gzil F, Cammelli M, Lefeve C, Pachoud B, Ville I. Person-centredness: conceptual and historical perspectives. Disabil Rehabil. 2007;29(20-21):1555-1565. doi:10.1080/0963828070161 8661 
25. Rodriguez-Osorio CA, Dominguez-Cherit G. Medical decision making: paternalism versus patient-centered (autonomous) care. Curr Opin Crit Care. 2008;14(6):708-713. doi:10.1097/MCC.0b013e328315a611

26. Epp TD. Person-centred dementia care: a vision to be refined. Can Alzheimer Dis Rev. 2003; April,14-18.

27. Talerico KA, O'Brien JA, Swafford KL. Person-centered care. An important approach for 21 st century health care. J Psychosoc Nurs Ment Health Serv. 2003;41(11):12-16.

28. Werner P, Koroknay V, Braun J, Cohen-Mansfield J. Individualized care alternatives used in the process of removing physical restraints in the nursing home. $J$ Am Geriatr Soc. 1994;42(3):321-325. doi:10.1111/j.1532-5415.1994.tb01759.x

29. Williams B, Cattell D, Greenwood M, LeFevre S, Murray I, Thomas P. Exploring "person-centredness": user perspectives on a model of social psychiatry. Health Soc Care Community. 1999;7(6):475-482.

30. Osborn Gould M. Resident-centered care. Heal Prog. 2001;82(6):5658.

31. Ashburner C, Meyer J, Johnson B, Smith C. Using action research to address loss of personhood in a continuing care setting. Illness, Cris Loss. 2004;12(1):23-37. doi:10.1177/1054137303259739

32. Bergman-Evans B. Beyond the basics. Effects of the Eden alternative model on quality of life issues. J Gerontol Nurs. 2004;30(6):27-34. doi:10.3928/0098-9134-20040601-07

33. Tuckett AG. On paternalism, autonomy and best interests: telling the (competent) aged-care resident what they want to know. Int J Nurs Pract. 2006;12(3):166-173. doi:10.1111/ijn.2006.12.issue-3

34. Edvardsson D, Winblad B, Sandman P. Person-centred care of people with severe Alzheimer's disease: current status and ways forward. Lancet Neurol. 2008;7(4):362-367. doi:10.1016/S1474-4422(08) 70063-2

35. Yoon JY. Relationships among person-centered care, nursing home adjustment, and life satisfaction: a cross-sectional survey study. Int Psychogeriatr. 2018;30(10):1519-1530. doi:10.1017/S1041610218 000194

36. Boumans N, Berkhout A, Landeweerd A. Effects of resident-oriented care on quality of care, wellbeing and satisfaction with care. Scand J Caring Sci. 2005;19(3):240-250. doi:10.1111/scs.2005.19.issue-3

37. McMillan SS, Kendall E, Sav A, et al. Patient-centered approaches to health care. Med Care Res Rev. 2013;70(6):567-596. doi:10.1177/ 1077558713496318

38. Berkhout JMB, Boumans PG, Nijhuis JN, Van Breukelen G, Huijer Abu-saad H. Effects of resident-oriented care on job characteristics of nursing caregivers. Work Stress. 2003;17(4):337-353. doi:10.1080/ 02678370310001647645

39. Pol-Grevelink A, Jukema JS, Smits CHM. Person-centred care and job satisfaction of caregivers in nursing homes: a systematic review of the impact of different forms of person-centred care on various dimensions of job satisfaction. Int J Geriatr Psychiatry. 2012;27 (3):219-229. doi:10.1002/gps.2716

40. Røen I, Kirkevold Ø, Testad I, Selbæk G, Engedal K, Bergh S. Person-centered care in Norwegian nursing homes and its relation to organizational factors and staff characteristics: a cross-sectional survey. Int Psychogeriatr. 2018;30(9):1279-1290. doi:10.1017/ S1041610217002708

41. WHO, The World Health Report 2000 - Health Systems: Improving Performance. WHO; 2013. Available from: https://www.who.int/whr/ 2000/en/. Accessed July 29, 2019.

42. The Health Foundation. Person-centred Care Made Simple What Everyone Should Know about Person-centred Care. London; 2016. Available from: https://www.health.org.uk/sites/default/files/ PersonCentredCareMadeSimple.pdf. Accessed July 29, 2019.

43. Harding E, Wait S, Scrutton $J$ The state of play in person-centred care: report summary. 2015. Available from: http://www.healthpolicy partnership.com/person-centred-care/http://personcentredcare.health. org.uk/around-the-world. Accessed July 29, 2019.
44. Crossing the quality chasm. Available from: http://www.nationalaca demies.org/hmd/ /media/Files/ReportFiles/2001/Crossing-theQuality-Chasm/QualityChasm2001reportbrief.pdf. Accessed July 29, 2019.

45. Lawton MP, Nahemow L. Ecology and the aging process. In: Eisdorfer C, Lawton MP (Eds.). The Psychology of Adult Development and Aging. Washington, DC, US: American Psychological Association; 1973: 619-674.

46. Langer EJ, Rodin J. The effects of choice and enhanced personal responsibility for the aged: a field experiment in an institutional setting. J Pers Soc Psychol. 1976;34(2):191-198. doi:10.1037/00223514.34.2.191

47. Pavlish C, Brown-Saltzman K, Hersh M, Shirk M, Rounkle AM. Nursing priorities, actions, and regrets for ethical situations in clinical practice. J Nurs Scholarsh. 2011;43(4):385-395. doi:10.1111/j.15475069.2011.01422.x

48. Fernández Ballesteros R, Benetos A, Robine J-M. The Cambridge Handbook of Successful Aging. ISBN 9781107162259: New York: Cambridge University Press; 2018:384-400.

49. Schaie KW, Klaus W. Aging Independently : Living Arrangements and Mobility. New York: Springer; 2003:355.

50. Wahl H-W, Schilling O, Oswald F, Iwarsson S. The home environment and quality of life-related outcomes in advanced old age: findings of the ENABLE-AGE project. Eur J Ageing. 2009;6(2):101111. doi:10.1007/s10433-009-0114-z

51. America I of M (US) C on Q of HC in. Crossing the Quality Chasm: A New Health System for the 21st Century. Washington (DC): National Academies Press (US); 2001.

52. Brownie S, Nancarrow S. Effects of person-centered care on residents and staff in aged-care facilities: a systematic review. Clin Interv Aging. 2013;8:1. doi:10.2147/CIA.S38589

53. Burgio LD, Allen-burge R, Roth DL, et al. Come talk with me : improving communication between nursing assistants and nursing home residents during care routines. Gerontologist. 2001;41(4):449_ 460. doi:10.1093/geront/41.1.24

54. Beck C, Ortigara A, Mercer S, Shue V. Enabling and Empowering Certified Nursing Assistants for Quality dementia care. Int J Geriatr Psychiatry. 1999:197-211.

55. McCallion P, Toseland RW, Freeman K. An evaluation of a family visit education program. J Am Geriatr Soc. 1999;47(2):203-214. doi:10.1111/j.1532-5415.1999.tb04579.x

56. Allen-Burge R, Burgio LD, Bourgeois MS, Sims R, Nunnikhoven J. Increasing communication among nursing home residents. J Clin Geropsychol. 2001;7(3):213-230. doi:10.1023/A:1011343212424

57. Ribera Casado JM, Bustillos A, Guerra Vaquero AI, Huici Casal C, Fernández-Ballesteros R. ¿Se discrimina a los mayores en función de su edad? Visión del profesional. Rev Esp Geriatr Gerontol. 2016;51 (5):270-275. doi:10.1016/j.regg.2016.03.005

58. Levy B, Langer E. Aging free from negative stereotypes: successful memory in China and among the American deaf. J Pers Soc Psychol. 1994;66(6):989-997. doi:10.1037/0022-3514.66.6.989

59. WHO. World Report on Ageing and Health 2015. Geneva. Switzerland. WHO; 2017. Available from: https:/www.who.int/age ing/events/world-report-2015-launch/en/. Accessed July 29, 2019.

60. Fernández-Ballesteros R, Caprara M, Schettini R, et al. Effects of university programs for older adults: changes in cultural and group stereotype, self-perception of aging, and emotional balance. Educ Gerontol. 2013;39(2):119-131. doi:10.1080/03601277.2012. 699817

61. Martin G, Pear J. Behavior Modification : What It Is and How to Do It. Boston: Pearson Education/Allyn \& Bacon; 2011:462.

62. Eymard AS, Douglas DH. Ageism among health care providers and interventions to improve their attitudes toward older adults: an integrative review. J Gerontol Nurs. 2012;38(5):26-35. doi:10.3928/ 00989134-20120307-09 
63. Kotter-Grühn D, Hess TM. The impact of age stereotypes on selfperceptions of aging across the adult lifespan. J Gerontol B Psychol Sci Soc Sci. 2012;67(5):563-571. doi:10.1093/geronb/gbr153

64. Levy B. Stereotype embodiment: A psychosocial approach to aging. In: Current Directions in Psychological Science. Vol. 18 Sage Publications, Inc.Association for Psychological Science; 2009:332-336.

65. Fingerman KL, Berg CA, Smith J, Antonucci TC. Handbook of Lifespan Development. Springer. 905.

66. Levy BR, Banaji MR. Implicit ageism. In: Nelson TD, editor. Ageism: Stereotyping and Prejudice against Older Persons. Cambridge (MA, US): The MIT Press; 2002:49-75.

67. Fernández-Ballesteros R, Sánchez-Izquierdo $M$, Olmos R, et al. Development and validation of a paternalism \& autonomist care assessment. J Adv Nurs. 2019, in press.

68. Fernandez-Ballesteros R, Olmos R, Santacreu M, Bustillos A, Molina MA. The role of perceived discrimination on active aging. Arch Gerontol Geriatr. 2017;71:14-20. doi:10.1016/j.archger.2017.02.004

69. Fernández-Ballesteros R. Sistema De Evaluación De Residencias De Ancianos: SERA (SID). INSERSO. Madrid: Instituto Nacional de Servicios Sociales; 1995:352.

70. Moos RH, Lemke S. Evaluating Residential Facilities: The Multiphasic Environmental Assessment Procedure. - PsycNET. CA, US: Sage Publications, Inc.; 1996.

71. Fiske ST, Cuddy AJC, Glick P, Xu J. A model of (often mixed) stereotype content: competence and warmth respectively follow from perceived status and competition. J Pers Soc Psychol. 2002;82 (6):878-902. doi:10.1037/0022-3514.82.6.878

72. Fernández-Ballesteros R, Sánchez-Izquierdo M, Olmos R, Huici C, Ribera Casado JM, Cruz Jentoft A. Paternalism vs. autonomy: are they alternative types of formal care? Front Psychol. 2019;10. doi:10.3389/fpsyg.2019.00038
73. Burnes D, Sheppard C, Henderson CR, et al. Interventions to reduce ageism against older adults: a systematic review and meta-analysis. $\mathrm{Am}$ J Public Health. 2019;109(8):e1-e9. doi:10.2105/AJPH.2019.305123

74. Weiner AS, Ronch JL (Eds). Culture Change in Long-term Care. New York: Haworth Social Work Practice Press. .

75. Chang Y-P, Li J, Porock D. The effect on nursing home resident outcomes of creating a household within a traditional structure. J Am Med Dir Assoc. 2013;14(4):293-299. doi:10.1016/j.jamda.2013.01.013

76. Grant LA, Fund C. Culture Change in a For-Profit-Nursing Home Chain: An Evaluation. Commonwealth Fund; 2008. Available from: https://www.commonwealthfund.org/publications/fund-reports/2008/ feb/culture-change-profit-nursing-home-chain-evaluation. Accessed July 29, 2019.

77. Kihlgren M, Hallgren A, Norberg A, Bråne G, Karlsson I. Effects of the training of integrity-promoting care on the interaction at a longterm ward. Scand J Caring Sci. 1990;4(1):21-28. doi:10.1111/j.14716712.1990.tb00003.x

78. Kihlgren M, Lindsten IG, Norberg A, Karlsson I. The content of the oral daily reports at a long-term ward before and after staff training in integrity promoting care. Scand J Caring Sci. 1992;6(2):105-112. doi:10.1111/j.1471-6712.1992.tb00132.x

79. Kihlgren M, Kuremyr D, Norberg A, et al. Nurse-patient interaction after training in integrity promoting care at a long-term ward: analysis of video-recorded morning care sessions. Int J Nurs Stud. 1993;30 (1):1-13. doi:10.1016/0020-7489(93)90088-C

80. Salas E, DiazGranados D, Klein C, et al. Does team training improve team performance? a meta-analysis. Hum Factors J Hum Factors Ergon Soc. 2008;50(6):903-933. doi:10.1518/001872008X375009

81. Salas E, Weaver SJ, DiazGranados D, Lyons R, King H. Sounding the call for team training in health care: some insights and warnings. Acad Med. 2009;84(Supplement):S128-S131. doi:10.1097/ACM.0b013e318 $1 \mathrm{~b} 37 \mathrm{~d} 48$
Clinical Interventions in Aging

\section{Publish your work in this journal}

Clinical Interventions in Aging is an international, peer-reviewed journal focusing on evidence-based reports on the value or lack thereof of treatments intended to prevent or delay the onset of maladaptive correlates of aging in human beings. This journal is indexed on PubMed Central, MedLine, CAS, Scopus and the Elsevier

\section{Dovepress}

Bibliographic databases. The manuscript management system is completely online and includes a very quick and fair peer-review system, which is all easy to use. Visit http://www.dovepress.com/ testimonials.php to read real quotes from published authors. 